\title{
Change the rules for food additives
}

\author{
John Ashby
}

The Delaney clause, embodied in US legislation in 1958, prohibits the addition to food of any level of any carcinogen. This position cannot be sustained in the face of progress in understanding chemically induced cancer.

THE Delaney clause of the US Food Additives Amendment, 1958, states that "no [food] additive shall be deemed safe if it is found to induce cancer when ingested by man or animals...". According to its author, Representative James J. Delaney of New York, "carcinogens are subtle, stealthy, sinister saboteurs of life. They have no place in our food chain"1.

In 1988, the US Environmental Protection Agency began granting tolerances under de minimis exceptions to the clause, permitting a risk of one case of cancer in 1 million people during 70 years, if within the context of a favourable risk/benefit assessment. But the US courts have decided that no such exceptions can be granted unless the Delaney clause is changed by Congress ${ }^{2}$. The main scientific obstacles to change appear to be the assumptions implicit in the clause that all rodent carcinogens pose an equal hazard to humans, and that one molecule of any carcinogen, acting on a single cell, can induce tumours (see the Correspondence from $\mathrm{T}$. Jukes on page 580).

Legislation to prevent addition of carcinogens to food seems to be an intrinsically good thing. But progress over the past 36 years in our understanding of chemically induced carcinogenesis has been so dramatic as to call into serious question the validity of the Delaney clause. Cicero stated that law is founded in nature, not in opinion - but the Delaney clause is founded in little more than opinion. Nonetheless, I believe, like Delaney, that there should be means to prevent the trivial addition to foods of carcinogens that are expected to cause cancer in humans.

In 1958, there were only a few known human carcinogens, such as benzidine, and a few known rodent carcinogens, such as benzanthracene. The bioassay protocols used at the time recognized only clear, potent carcinogens. Within that context, legislation to prevent addition to food of such "sinister saboteurs of life" seemed to be justified. James and Elizabeth Miller then discovered that carcinogens, or their metabolites, are reactive to cellular macromolecules such as DNA, and Bruce Ames and subsequent workers established that they were also mutagenic (genotoxic) across phyla - carcinogens were mutagens with recognizable reactive centres within their chemical stuctures. Growing concern in the mid-1970s about the true prevalence of carcinogens in the environment led the US National Cancer Institute and National Toxicology Program (NTP) to initiate a series of detailed cancer bioassays in rats and mice of chemicals of environmental importance.

To date, results from more than 300 chemicals have been published, only a third of which show no evidence of rodent carcinogenicity. About one half of the new carcinogens were of the classical genotoxic type - active in several tissues and/or in both species, and mutagenic. A further 60 were uniquely active at single sites of only one species, and then often in only one gender. In addition, the apparently benign chemical structures and the absence of mutagenicity for most of these 60 carcinogens are wholly inconsistent with a genotoxic mechanism of carcinogenic action ${ }^{3}$. These presumed non-genotoxic carcinogens are thought to increase the tumour incidence in rodents by physiological mechanisms associated with chemically induced cell division. The induction of renal tumours uniquely in male rats by the renal mitogen limonene (a constituent of limes and lemons) is the best studied non-genotoxic carcinogen (see ref. 4 for a review).

In the absence of the unifying hypothesis of DNA reactivity, any hazard these non-genotoxic carcinogens might pose to humans must be individually established, not assumed. Lemon juice cannot travel on the coat-tails of benzidine. Further, if the 100 or so non-carcinogens defined by the NTP were to be submitted to further bioassays in different species or strains of test animal, then many would probably show some non-genotoxic carcinogenic properties $^{3}$. The term 'rodent carcinogen' has therefore drifted far away from the threat envisaged by Delaney.

The case of the flavouring agent and perfume additive benzyl acetate illustrates the point. In 1986, the NTP reported benzyl acetate to be carcinogenic when administered by oral gavage (intubation) in corn oil. Tumours were observed in the male rat pancreas and in the stomach and liver of both sexes of mice ${ }^{5}$. Benzyl acetate has a non-reactive chemical structure and is non-genotoxic ${ }^{6}$. The NTP expressed concern that the corn oil vehicle may itself have caused the pancreatic tumours, and that local irritation of the stomach induced by high local concentrations of the chemical might have caused the stomach tumours. The NTP therefore retested it in a feeding study, in which it was found to be uniformly non-carcinogenic to rats and mices.

What is one to make of such conflicts of data? First, unqualified use of the term 'rodent carcinogen' is an insecure basis for legislation. Second, physiological disturbances caused by maximum-tolerated doses of chemicals may lead to isolated instances of rodent carcinogenesis of dubious or no relevance to humans ${ }^{7}$. Third, a rational application of the Delaney clause to chemicals such as benzyl acetate is impossible; the overwhelming evidence is that benzyl acetate will be noncarcinogenic to humans, yet it remains a 'carcinogen' for legal purposes.

There are various ways in which a newly discovered rodent carcinogen can be classified as genotoxic or non-genotoxic ${ }^{8,9}$. In the case of a probable genotoxic carcinogen, benefit/risk assessments can lead to de minimis exceptions in appropriate cases. In the case of a probable nongenotoxic rodent carcinogen, additional data, or data on analogues, can indicate if the carcinogenic effect is rodent-specific or if a hazard to humans exists. Many non-genotoxic carcinogens will be found on this basis to pose no risk to humans; for example, corn oil, lemon juice, oxazepam and benzyl acetate - but some might pose a hazard; for example, dioxin (TCDD).

Given that many rodent carcinogens are now known to be ubiquitous in foods and the environment, what is needed is a legal framework sensitive to advances in understanding of chemical carcinogenicity. This will allow the rational use of all relevant general information on chemical carcinogenicity, coupled to that for the particular agent under study, leading to an estimate of carcinogenic hazard (or lack of it) to humans. Such an integrated process should be the key to effective human health protection, not only for food additives, but for pesticide residues, therapeutic drugs and environmental chemicals. $\square$

John Ashby is at Zeneca's Central Toxicology Laboratory, Alderley Park, Cheshire SK10 4TJ, UK.

\footnotetext{
1. Jukes, T. H Prev. Med. 2, 133-139 (1972).

2. Nature 363, 287 (1993)

3. Ashby, J. \& Purchase, I. F. H. Mutagenesis 8, 489-493 (1993)

4. Swenberg. J. A. Environ. Hith Perspect. 101 Suppl. 6 39 44; Suppl. 5 (1993)

5. NTP Technical Reports, 250 (1986); 431 (1993)

6. Ashby, J. Mutat. Res. 306, 107-109 (1994).

7. Ames, B. N. \& Gold, L. S. Science 249, 970-971(1990)

7. Ames, B. N. \& Gold, L. S. Science 249, 970-971(19)

9. Ashby, J. \& Morrod, R. S. Nature 352, 185-186 (1991).
} 